\title{
Involuntary attention and brightness contrast
}

\author{
William Prinzmetal, Virginia Long, and James Leonhardt \\ University of California, Berkeley, California
}

\begin{abstract}
Carrasco, Ling, and Read (2004) reported that involuntary attention increased perceived contrast. We replicated Carrasco et al. and then tested an alternative hypothesis: With stimuli near threshold, a peripheral cue biased observers to believe a stimulus had been presented in the cued location. Consistent with this hypothesis, the effect disappeared when we used higher-contrast stimuli. We further tested the guessing-bias hypothesis in three ways: (1) In a detection experiment, the cue affected bias, but did not increase $d^{\prime} ;$ (2) when the cue followed the stimulus, we obtained the same results as when the cue preceded the stimulus; (3) in one experiment, some trials contained no stimulus, yet observers responded that the cued blank stimulus had higher contrast than the uncued blank stimulus. The results suggest that the effects of a noninformative peripheral cue are best described in terms of nonperceptual biases.
\end{abstract}

Does attention change the apparent contrast of a stimulus? The issue of the effect of attention on the apparent strength (e.g., brightness or contrast) of a stimulus is one with its roots in the nineteenth century. For example, James believed that attention increased sensory impressions, commenting, "it must be admitted that to some extent the relative intensity of two sensations may be changed when one of them is attended to and the other is not ... in listening for certain notes in a chord, the one we attend to sounds probably a little more loud ..." (James, 1890, p. 425). ${ }^{1}$ Fechner, on the other hand, vehemently denied that attention increases the intensity of a stimulus, famously stating, "A gray paper appears to us no lighter, the pendulum-beat of a clock no louder, no matter how much we increase the strain of our attention upon them" (James, 1890, p. 426). Ebbinghaus (1908, p. 90) took a middle position: Attention would increase the intensity of a faint stimulus. For example, the ticking of a watch would go unnoticed unless one paid attention to it.

In the area of brightness contrast, more recent experimental work on this problem has yielded three divergent claims: (1) Attention decreases contrast (Tsal, Shalev, Zakay, \& Lubow, 1994); (2) attention increases neither contrast nor brightness, but makes perception more veridical (Prinzmetal, Nwachuku, Bodanski, Blumenfeld, \& Shimizu, 1997); (3) attention increases contrast (Carrasco, Ling, \& Read, 2004). The goal of the experiments reported here is to examine the claim by Carrasco et al. that involuntary attention increases the phenomenal contrast of a stimulus.

Tsal et al. (1994) performed the first experiments that directly manipulated attention to determine the effect of attention on phenomenal contrast. They conducted two kinds of experiments; in the first set, they manipulated attention with a spatial cue that indicated the most likely lo- cation of the stimulus (e.g., Posner, 1980; Posner, Snyder, \& Davidson, 1980). Since the cue was informative as to the stimulus location, this is a manipulation of voluntary attention (Prinzmetal, McCool, \& Park, 2005). In several experiments they found when the stimulus was in the cued location, it was judged to have less contrast than when it was in a uncued location. They also manipulated involuntary attention with the simultaneous presentation of two stimuli, asking which stimulus was brighter. This paradigm was identical to the one used by Carrasco et al. (2004); we will discuss Tsal et al.'s results using this second paradigm later. Tsal et al. concluded that attention (whether voluntary or involuntary) decreased brightness contrast.

In a series of 8 experiments, Prinzmetal et al. (1997) examined the effect of goal directed or voluntary attention on brightness and contrast. The critical task was to indicate the brightness of a gray square on either a white or black background. Observers responded in one of three ways: (1) by selecting a location on a palette that went from black to white; (2) by selecting one of five gray samples that matched the target in brightness; or (3) by verbally responding with a number from 1 to 5 to indicate the brightness of the target. (The latter method was used by Tsal et al., 1994.) Two different dual-task manipulations of attention were investigated. The critical task was always to indicate the brightness of the gray target. The other task was a letter identification task. In one manipulation of attention, the letters were either near the gray target or far from the gray target. The assumption was that it is easier to attend to two objects that are near each other than far from each other (Hoffman, Nelson, \& Houck, 1983). The second manipulation of attention was that the gray target and letters either came on simultaneously or successively. The assumption was that simultaneously processing the stimuli from two tasks demands more attention than pro-

W. Prinzmetal, wprinz@berkeley.edu 
cessing the information from one task at a time. Hence, in the simultaneous condition, observers would have fewer perceptual resources available for the brightness judgment task (Gardner, 1973; Hoffman, 1979; Prinzmetal \& Banks, 1983). All of the attention manipulations and methods of measuring brightness had the same result: Attention increased the accuracy of observers' judgments, but it did not systematically change the brightness or contrast.

Prinzmetal et al. (1997) also investigated the discrepancy between their results and the results of Tsal et al. (1994) using an informative spatial cue. Prinzmetal et al. (1997) were able to replicate the previous results and demonstrate that the results of Tsal et al. were due to the manner that observers responded and the particular training regime used by Tsal et al. ${ }^{2}$

Prinzmetal and his colleagues investigated the effect of attention on a wide variety of stimulus properties including hue (color), line orientation, line length, spatial frequency, and location (Prinzmetal, 2005; Prinzmetal, Amiri, Allen, \& Edwards, 1998; Prinzmetal \& Wilson, 1997). They used a variety of manipulations of attention and response methods. In nearly every case, attention had a clear effect on performance: Attention made observers more accurate, but it did not cause any shifts in perceptual judgments. As we will discuss later, a critical aspect of all of these experiments was that the stimuli were above threshold and there was no location uncertainty. ${ }^{3}$

The specific purpose of the present research is to examine a recent report by Carrasco et al. (2004). In contrast to Prinzmetal et al. (1997), who found that voluntary attention increased the accuracy of judgments of brightness or contrast, and Tsal et al. (1994), who found that attention decreased perceived contrast, Carrasco et al. reported that automatic or involuntary attention increased perceived contrast. In Carrasco et al., on each trial two Gabor stimuli were briefly presented. The stimuli were either oriented $45^{\circ}$ clockwise or $45^{\circ}$ counterclockwise from vertical. The task in the two main experiments was to indicate which Gabor stimulus had the higher contrast and to indicate the orientation of that stimulus. One Gabor stimulus (the standard) always had the same contrast. The other stimulus (the test) varied in contrast from less than to greater than the standard.

Automatic or involuntary attention was manipulated by presenting a cue (i.e., black dot) just above one of the Gabor stimuli, $116 \mathrm{msec}$ before the stimulus appeared (see Figure 1). This is a manipulation of involuntary attention, because the location of the cue was uncorrelated with the location of the higher-contrast Gabor stimulus. The results were that the psychometric function was shifted for the cued Gabor stimulus relative to the uncued stimulus; that is, there was a tendency to select the cued stimulus as the one with higher contrast. The authors used two contrast ranges, one near threshold (Experiment 1) and one with slightly higher contrast (Experiment 2), in two otherwise identical experiments. In both experiments, they found the same results: Attention increased the tendency for observers to select the cued stimulus location as having higher contrast.

Schneider (2006), using a wide range of brightness and contrast levels, replicated Carrasco et al. (2004), but only when the stimuli were very near (or below) threshold. Schneider used dots rather than Gabor stimuli, and the cue was an annulus surrounding the dot. Schneider argued that involuntary attention improves the detection threshold, making it more likely that a low-contrast stimulus would

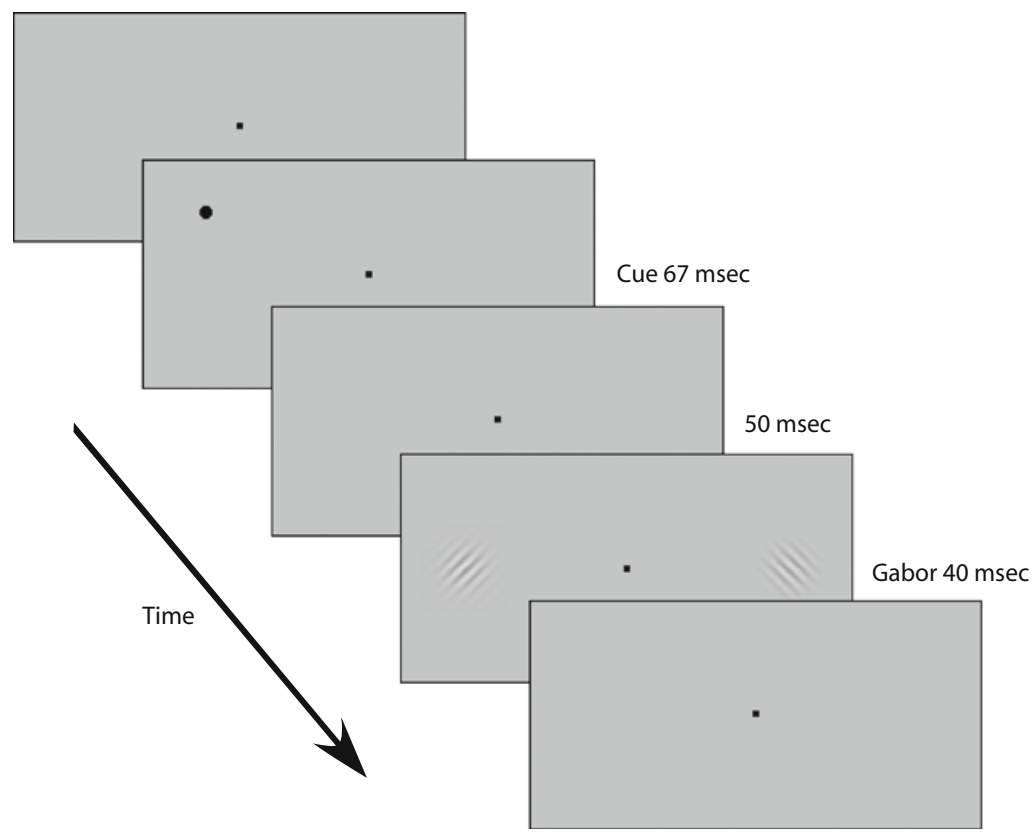

Figure 1. The sequence of events in Experiment 1. 
be detected. Thus the cued stimulus would be more likely to be detected than would the uncued stimulus. Hence the cued stimulus would be judged to have higher contrast than the uncued and undetected stimulus. But Schneider claimed that attention did not increase contrast, per se. Recently, Schneider and Komlos (in press) replicated Carrasco et al. using Gabor patterns identical to Carrasco et al.'s. They also found that the Carrasco et al. effect got smaller as the stimulus contrast decreased.

We began with an experiment that was very similar to their experiments. Carrasco and her colleagues kindly sent us the program that they had used to run their Experiments 1 and 2 . We were able to create our stimuli by running their program (their Experiment 2) and taking a screen snapshot of their standard stimuli. We then very carefully crafted our Experiment 1 to be similar to their Experiment 2 (higher contrast stimuli). In our Experiment 1 , below, we replicated their results.

In Experiment 2, we increased the contrast of the stimulus, and consistent with Schneider (2006) and Schneider and Komlos (in press), the effect of the noninformative spatial cue was greatly reduced. This pair of findings is in agreement with the hypothesis that involuntary attention increases contrast, but only for low-contrast stimuli. We believed it was important to replicate the results of Carrasco et al. (2004) and Schneider, because without replication we could not be sure that we had captured the essential features of their effects.

Having replicated Carrasco et al. (2004) and Schneider (2006), we tested an alternative explanation based on a guessing bias. With low-contrast stimuli, observers might not perceive the Gabor stimuli on some trials, but they might assume that there was something in the cued location. They would do this because the cue and the Gabor stimulus are both visual transients. If observers believe that something was in the cued location, logically, that something must have had higher contrast than did the uncued location, where nothing was perceived. Note that in order for such a guessing bias to affect performance, this bias effect need occur only on a few trials.

We tested this alternative hypothesis in three ways. In Experiment 3 we used a detection task. If involuntary attention increases contrast, it should increase $d^{\prime}$ for cued stimuli. If the spatial cue is having its effect by changing bias, it should not increase $d^{\prime}$ but rather change the observer's criterion, so that observers are more likely to make false alarms. In Experiment 4, we reversed the temporal order of the Gabor stimuli and the cue. If the cue has its effect on perceptual processes, it should not influence performance if it comes after the stimulus (for similar arguments, see Liu, Pestilli, \& Carrasco, 2005; Prinzmetal et al., 2005; Woodman, Vecera, \& Luck, 2003). On the other hand, if the same effect was obtained when the cue appeared after the stimulus, it would suggest a postperceptual mechanism. In the final experiment, unbeknownst to observers, on $20 \%$ of the trials there was no stimulus in either the cued or the uncued location. Increasing the contrast gain on a blank location should not result in the perception of a Gabor stimulus of higher contrast. Therefore, if the previous effects were due to bias, observers should choose the cued blank location as having more contrast than the uncued blank location.

\section{EXPERIMENT 1}

The purpose of Experiments 1 and 2 was to replicate the effects of Carrasco et al. (2004) and Schneider (2006). Experiments in different labs are never precisely the same in every detail. Without replicating the previous findings, we cannot be sure that we have captured the essential features of the previous research. Hence our comparisons with Carrasco et al. and Schneider began with replications of their results.

The goal of Experiment 1 was to conduct a replication of Carrasco et al. (2004) that was sufficiently similar to their Experiment 2 so as to yield the same results as they obtained. Our standard stimulus was, in fact, created from their computer program, as described below.

\section{Method}

Procedure. The procedure is illustrated in Figure 1. A fixation dot was present throughout a block of trials. On each trial, a cue (black dot) was presented for $67 \mathrm{msec}$. On one third of the trials, the left location was cued, on one third the right location was cued, and on one third the cue replaced the fixation dot (center or neutral cue). After an interval of $50 \mathrm{msec}$, two Gabor stimuli were presented for $40 \mathrm{msec}$, one on each side of the fixation point. Subsequent to the presentation of the Gabor stimuli, the observer responded as to the location and orientation of the Gabor stimulus with the higher contrast. Observers were instructed to indicate the orientation of the Gabor with the higher contrast by pressing one of 4 keys on the keyboard: " $Z$ " or " $X$ " if the left Gabor stimulus had higher contrast; "?" or "/" if the right Gabor stimulus had higher contrast. The keys " $Z$ " and "." were used if the stimulus was rotated counterclockwise, and " $X$ " and "/" were for clockwise rotation. Thus, as with Carrasco et al. (2004), one buttonpress indicated the orientation and location of the stimulus with higher contrast. The response assignment was identical to Carrasco et al. Observers were told that there was no relation between the cue and the stimulus with the higher contrast.

We were not clear as to one aspect of the procedure of Carrasco et al. (2004). Their article indicated that observers had to respond within $1 \mathrm{sec}$, whereas the software provided to us did not have any fixed response window. Also, the article did not mention the consequences of not responding quickly enough; we therefore ran two groups of observers. One group of observers was urged to take their time and be as accurate as possible. The other group had to respond within $1 \mathrm{sec}$; if they did not, the computer emitted a "beep" tone when they eventually did. Observers exceeded the time limit on only $1.6 \%$ of trials. At the end of each block, observers were given the percentage of correct responses on the orientation judgment, but no other feedback was given. For the no-time-limit group, the experiment took about $50 \mathrm{~min}$; for the time-limit group, it took about $30 \mathrm{~min}$.

Each observer was given a minimum of 2 practice blocks of 48 trials. If their performance was below $50 \%$ on the orientation judgment, they were given additional blocks of practice. Data was collected in 4 blocks of 192 trials per block.

Stimuli. The stimuli were presented on a 15-in. monitor controlled by a Macintosh G3 computer ${ }^{4}$ in a dark room. The Gabor stimuli were created in the following way: We modified the software of Carrasco et al.'s (2004) computer program so that each Gabor had the contrast of the standard. We then took a screen snapshot (using Snapz computer program) of the standard ${ }^{5}$ We created 4 test stimuli by taking the standard and decreasing and/or increasing the contrast of the standard. Thus there were 2 test stimuli above and 2 below the contrast of the standard. The frequency was 7 cycles/deg. 
Table 1

Contrast of Gabor Stimuli (in $\mathrm{cd} / \mathrm{m}^{2}$ and in Barlow Units)

\begin{tabular}{|c|c|c|c|c|c|c|c|c|}
\hline & \multicolumn{4}{|c|}{ Experiment 1} & \multicolumn{4}{|c|}{ Experiment 2} \\
\hline & $\begin{array}{c}\text { Peak } \\
\text { (Michelson) }\end{array}$ & $\begin{array}{l}\text { High } \\
\text { Value }\end{array}$ & $\begin{array}{l}\text { Low } \\
\text { Value }\end{array}$ & $\begin{array}{c}\text { Barlow } \\
\text { Units }\end{array}$ & $\begin{array}{c}\text { Peak } \\
\text { (Michelson) }\end{array}$ & $\begin{array}{l}\text { High } \\
\text { Value }\end{array}$ & $\begin{array}{l}\text { Low } \\
\text { Value }\end{array}$ & $\begin{array}{c}\text { Barlow } \\
\text { Units }\end{array}$ \\
\hline Test 1 & 0.17 & 14.68 & 10.48 & 17.69 & 0.41 & 19.20 & 8.02 & 25.71 \\
\hline Test 2 & 0.19 & 14.95 & 10.12 & 19.16 & 0.48 & 20.21 & 7.04 & 27.22 \\
\hline Standard & 0.23 & 15.65 & 9.85 & 20.54 & 0.57 & 22.93 & 6.30 & 28.89 \\
\hline Test 3 & 0.29 & 16.72 & 9.13 & 22.72 & 0.75 & 27.63 & 3.95 & 31.34 \\
\hline Test 4 & 0.37 & 18.25 & 8.31 & 25.04 & 0.98 & 35.42 & 0.37 & 34.26 \\
\hline
\end{tabular}

Note-The gray background of the monitor on which the stimuli appeared was $12.43 \mathrm{~cd} / \mathrm{m}^{2}$.

Table 1 describes the contrast of the 5 stimuli in several different ways. The luminance values of the highest and lowest pixels in the stimuli were based on measurements made with a spot photometer with the lighting at which the experiment was run, from the viewing distance of the observer. Michelson contrast (i.e., peak contrast) is based on these values. However, peak contrast is not a good measure of threshold performance with Gabor stimuli and other spatially finite stimuli (Watson, 2000) because peak contrast represents the contrast of only a few, highest contrast, pixels. Hence we also report the contrast in Barlow units. Barlow units are defined as follows:

$$
\mathrm{dBB}=10 * \log _{10}\left(\mathrm{CE} * 10^{6}\right) .
$$

$\mathrm{CE}$ is contrast energy, which is the squared contrast integrated over space and time (Watson, 2000; Watson \& Ahumada, 2005; Watson, Barlow, \& Robson, 1983). ${ }^{6}$ These units have been shown to be better predictors of threshold and detection performance than peak contrast (e.g., Watson, 2000; Watson \& Ahumada, 2005), and they have been used over a wide range of stimuli (e.g., Beard \& Ahumada, 1999). In addition to frequency, contrast, and eccentricity, performance is also related to other factors, such as the size of the Gaussian envelope and phase (Foley, Varadharajan, Koh, \& Farias, 2007). To provide an accurate description of the stimuli, Figure 2 plots the luminance profiles, in a direction orthogonal to the grating, of the highest and lowest contrast stimulus used in Experiment 1.

There were a few differences between their program and ours. They used 23 different test values; we only used 4 . Since most of their effect was when the test values were close in contrast to the standard value, we wanted to get as much data in that region as possible. We only used the high-frequency stimuli because their effect was largest with them. Because of a limitation in their software, to get enough distinct luminance values their stimuli were luminance-varying shades of green, whereas ours used shades of gray. Also, because of

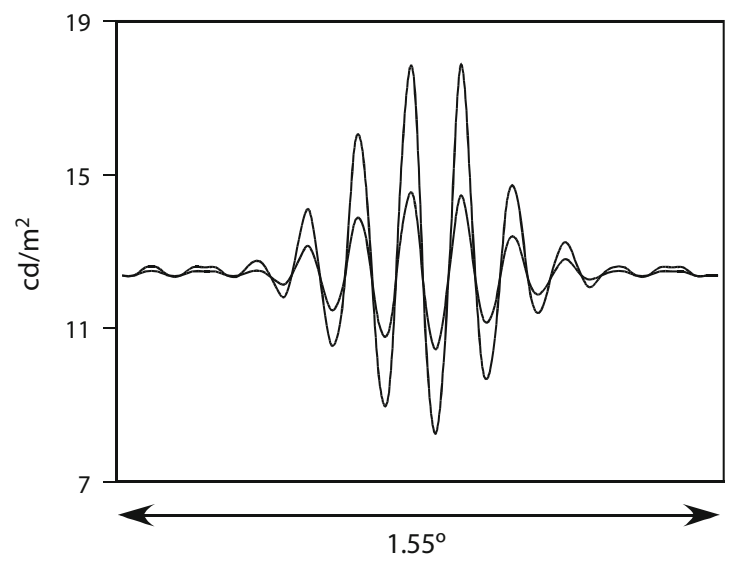

Figure 2. Luminance profile of highest and lowest contrast stimuli in Experiment 1. differences in monitors, our stimuli were somewhat higher in contrast (see Table 1) and higher in frequency (7 cycles/deg) than theirs were. In all other respects, we attempted to make our experiment as identical to theirs as possible. Nevertheless, because of the difference in stimuli and procedure, it was necessary to replicate their results.

Observers were seated $100 \mathrm{~cm}$ from the monitor and their heads were stabilized with chinrests. At this viewing distance, the distance between the centers of the Gabor stimuli subtended $3.7^{\circ}$ of visual angle. The other components of the stimuli (e.g., fixation point and cue) were identical, so that when we superimposed screen snapshots of their stimuli and our stimuli, we could detect no differences.

Observers. Eight observers, ages 18 to 22, participated in each group. In all the experiments reported here, approximately one half of the observers were male and one half female. Observers were recruited from the research participant pool of the Psychology Department at the University of California, Berkeley, and were given course credit for their participation.

\section{Results and Discussion}

The proportion of trials on which observers responded that the test Gabor stimulus had greater contrast than the standard stimulus is plotted in Figure 3 as a function of which item was cued (center, standard, or test). Confidence intervals were calculated as described by Loftus and Masson (1994) for a mixed design experiment. When the test item was cued, observers were more likely to respond that it had higher contrast than when the standard item was cued (Figure 1). We conducted an ANOVA with cue type, test contrast value, and group (time limit vs. no time limit) as factors. Note that the significance level of all ANOVAs has been corrected for violations of variance-covariance homogeneity with the Greenhouse-Geisser method (although uncorrected $d f$ s are reported). There was a significant effect of the cue $[F(2,28)=3.13, p<.01]$, indicating that observers were more likely to respond with the cued location. There was also a significant interaction of group (time limit, no time limit) and which test stimulus was presented $[F(3,42)=3.68, p<.05]$. Essentially, the notime-limit group had a steeper psychometric function (i.e., performed better) than the time-limit group.

Not including the trials with a center cue, observers had a strong tendency to respond with the cued location (whether standard or test). In the time-limit group, 7 of the $8 \mathrm{ob}-$ servers responded with the cued item more than $50 \%$ of the time $(M=60.0 \%)$. In the no-time-limit group, all of the observers responded more than $50 \%$ of the time that the cued location was the location with the higher contrast $(M=59.0 \%)$.

In summary, our results were similar to those of Carrasco et al. (2004). However, there was one more obser- 

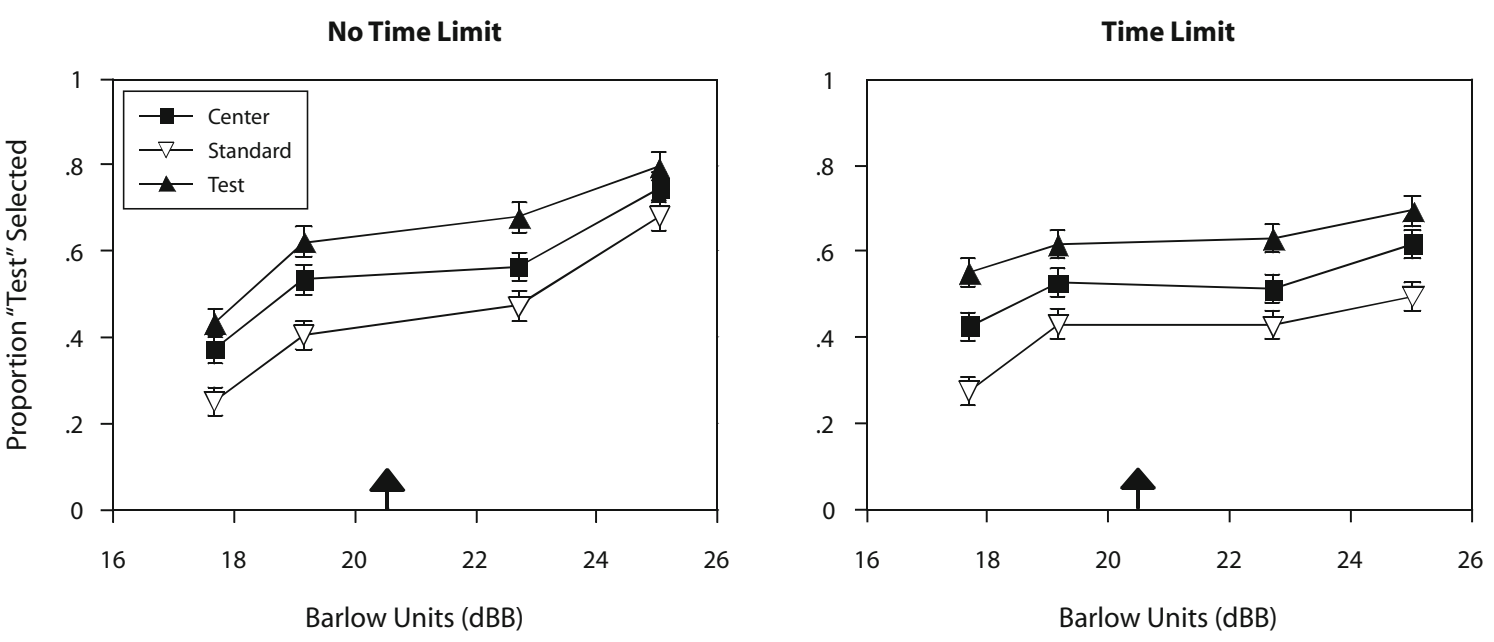

Figure 3. Experiment 1: The proportion of trials on which observers responded that the test stimulus had greater contrast than the standard stimulus, as a function of which item was cued (center, standard, or test). The arrows mark the contrast of the standard.

vation we found curious. We compared the accuracy of orientation judgments when the higher contrast item was cued and when the lower contrast item was cued (see Table 2). A correct response would be the orientation of the higher contrast item. When the location judgments are incorrect, the orientation judgments should be at chance (i.e., observers are responding on the basis of the wrong stimulus). When observers got the location incorrect, the orientation judgments were $47.2 \%$ correct when that item was cued and $51.9 \%$ correct when it was not cued. As expected, these values are close to chance.

When observers got the location correct, they got the orientation correct on an average of $76.9 \%$ of trials. One might expect that if observers attend to the cued location, they would be more accurate at the orientation judgments for targets in that location. However, this was not the case. For the time-limit group, observers were actually significantly less accurate on the orientation judgment when the higher contrast stimulus was cued than when

Table 2

Orientation Accuracy in Percent Correct

\begin{tabular}{|c|c|c|c|c|}
\hline & \multicolumn{4}{|c|}{ Location } \\
\hline & \multicolumn{2}{|c|}{ Correct } & \multicolumn{2}{|c|}{ Incorrect } \\
\hline & Cued & Uncued & Cued & Uncued \\
\hline \multicolumn{5}{|l|}{ Experiment 1} \\
\hline Time limit & 67.4 & 73.7 & 43.3 & 50.7 \\
\hline No time limit & 83.1 & 83.4 & 51.2 & 53.2 \\
\hline Experiment 2 & 90.2 & 89.2 & 51.2 & 49.8 \\
\hline \multicolumn{5}{|l|}{ Experiment 4A } \\
\hline $50 \mathrm{msec}$ postcue & 72.8 & 78.2 & 45.2 & 47.1 \\
\hline \multicolumn{5}{|l|}{ Experiment 4B } \\
\hline $50 \mathrm{msec}$ precue & 79.1 & * $\quad 84.0$ & 51.0 & 51.1 \\
\hline $300 \mathrm{msec}$ postcue & 82.6 & 83.2 & 48.6 & 50.1 \\
\hline \multicolumn{5}{|l|}{ Experiment 5} \\
\hline Gabor present & 66.3 & 66.3 & 47.6 & 49.6 \\
\hline
\end{tabular}

Note-In no case was there a significant advantage in orientation judgments for the cued over the uncued location. ${ }^{*} p<.05$, two-tailed. the other stimulus was cued $(67.4 \%$ vs. $73.7 \%$, respectively) $[t(7)=3.53, p<.05$, two-tailed]. For the group that did not have a time limit, there was no significant difference $(83.1 \%$ vs. $83.4 \%)[t(7)=0.30]$. Thus, the results on the orientation judgment task give no evidence that involuntary attention is operating in the sense of making perception more veridical. Carrasco et al. (2004) did not report the accuracy of orientation judgments. We had not predicted that observers would be worse on orientation judgments for the cued stimulus. A possible explanation of this effect is that on some trials, observers did not perceive a Gabor stimulus, but assumed that there was a Gabor stimulus in the cued location. Such a bias would lower the accuracy of orientation judgments in the cued location, because responses on these trials would be at chance. Whatever the reason for worse orientation performance for the stimulus at the cued location, one would expect that attention would increase the accuracy of orientation judgments (i.e., Prinzmetal et al., 1998), but this was not the case.

Experiment 1 replicated Carrasco et al. (2004) quite well, whether observers were put under time pressure or not. Schneider (2006) replicated Carrasco et al. only when the stimuli were low contrast. Schneider and Komlos (in press) found a larger effect as contrast was reduced. The stimuli in our Experiment 1 were near threshold. Some observers complained that they could not detect anything on some of the trials. In light of the fact that Schneider replicated Carrasco et al. only when the stimuli were near threshold, together with the present results, it seems likely that a prerequisite for this effect is to have the stimuli near threshold. However, Schneider's stimuli were not Gabor stimuli. Hence, in Experiment 2, we repeated Experiment 1 with higher contrast Gabor stimuli. Figure 4 compares the standard stimulus used in Experiments 1 and 2. (In Experiment 4, we verify that the stimuli were near threshold in Experiment 1 and above threshold in Experiment 2.) 


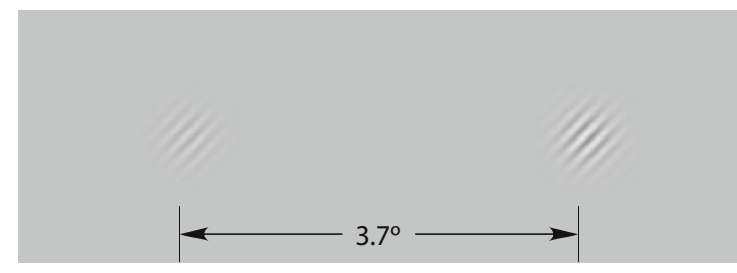

Figure 4. A comparison of the standard stimuli in Experiment 1 (left) and Experiment 2 (right). To view the stimuli as the observers did, adjust the viewing distance so that the distance between the Gabor stimuli subtends $3.7^{\circ}$ of visual angle.

\section{EXPERIMENT 2}

\section{Method}

Experiment 2 was an exact replication of the time-limited group in Experiment 1, except the contrast of the Gabor stimuli was increased (see Table 1 for the exact values). Eighteen observers were recruited from the UC Berkeley research participant pool as in Experiment 1.

\section{Results}

The results are shown in Figure 5. Unlike in Experiment 1 , the cue did not have a significant effect $[F(2,34)=$ $2.67, p>.10]$, nor did it interact with the test stimulus that was used $[F(6,102)=1.62, p>.10]$. Hence, like Schneider, we replicated Carrasco et al.'s (2004) results, but only with low-contrast stimuli.

We also examined the accuracy of orientation judgments (Table 2). There was no difference in the accuracy of orientation judgment when the stimuli were in the cued versus the uncued location.

\section{Discussion}

The purpose of Experiments 1 and 2 was to replicate the results of Carrasco et al. (2004) and of Schneider (2006). Experiment 1 replicated Carrasco et al. with low-contrast stimuli. Consistent with Schneider, when we increased the

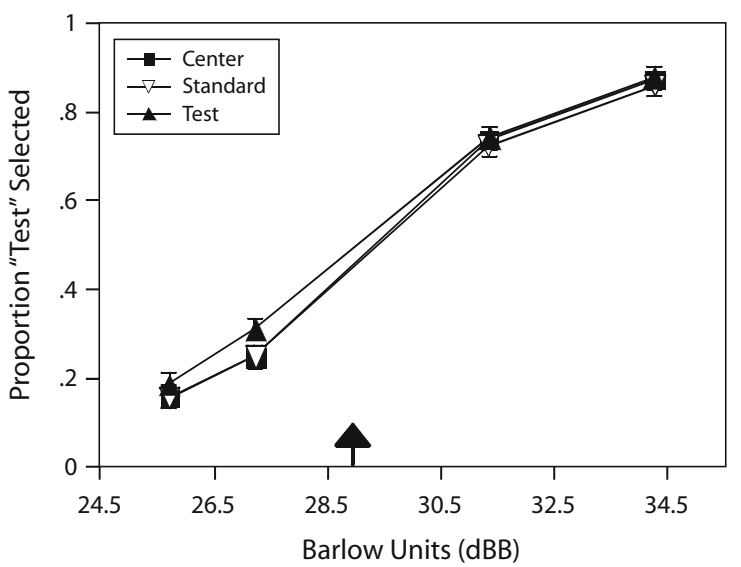

Figure 5. Experiment 2: The proportion of trials on which observers responded that the test stimulus had greater contrast than the standard stimulus, as a function of which item was cued (center, standard, or test). The arrow marks the contrast of the standard. contrast of the target stimulus, observers did not respond that the cued stimulus had greater contrast. Schneider replicated Carrasco et al. both with cues that consisted of a white annulus around the target and with a black annulus around the target. With both types of cues, they replicated Carrasco et al., but only when the stimuli were near threshold. Schneider's effect was larger with a white cue. With a black cue, the apparent contrast of the highcontrast stimuli was slightly reduced. He suggested that some of the reduction might have been due to a sensory interaction of the cue and target. Note however, with both white cues and black cues, Schneider replicated Carrasco et al., but the effect disappeared when the stimuli were higher in contrast, regardless of the cue.

Recently, Ling and Carrasco (2007) replicated the original Carrasco et al. (2004) study with both white and black cues. Their results were identical to Schneider's (2006) low-contrast conditions: They obtained similar results, regardless of the color of the cue. Ling and Carrasco did not vary contrast, however. Empirically, the results of all three studies are consistent in showing that, with stimuli near threshold, observers tend to chose the cued stimulus as having higher contrast, regardless of the color of the cue (Carrasco et al., 2004; Schneider, 2006; and the present experiments). Experiment 2 is consistent with Schneider in demonstrating that when we increased contrast, this effect disappeared. The remaining experiments investigated why the effect occurred with only low-contrast stimuli.

It could be argued that the reason that we did not obtain an effect on contrast judgments in Experiment 2 was that the discrimination was too easy, and hence involuntary attention could not affect performance. Note that this objection does not apply to Schneider (2006) or Schneider and Komlos (in press), who used a wide range of contrasts and backgrounds, so that discriminability and contrast were varied independently.

Given our results and Schneider's (2006), one might conclude that attention increases the apparent contrast of stimuli near threshold. There is an alternative explanation: It is possible that on trials in which observers did not perceive the Gabor stimuli, they assumed that a Gabor stimulus was in the cued location. They might make this assumption because they saw a flicker in that location (i.e., the cue) and therefore infer that there must have been something in that location. Alternatively, they might lower their detection threshold for the cued location rather than for the uncued location. Consequently, if they assumed there was "something" in the cued location, and they did not see anything in the noncued location, the logic of their response could be summarized as: "something has higher contrast than nothing." Such a bias would only occur with stimuli near threshold. Furthermore, if observers were asked which location had the least contrast, this bias would cause observers to choose the location that was not cued. In Carrasco et al.'s (2004) Experiment 3, this is precisely what was found.

\section{EXPERIMENT 3}

Experiment 3 was a detection experiment that directly tested the hypothesis that the results of Experiment 1 were 
not due to an increase in contrast, but rather to a bias to believe that something was presented in the cued location. For half the observers, we used the standard Gabor stimuli used in Experiment 1 (low contrast) and for the remaining observers, we used the standard from Experiment 2 (high contrast). In the left location, there was a $50 \%$ probability that a Gabor stimulus would be present, and on the right there was a $50 \%$ probability that a Gabor stimulus would be present. Thus there were four kinds of trials: (1) on $25 \%$ of the trials, there was no stimulus; (2) on $25 \%$ there was a stimulus only on the left; (3) on $25 \%$ there was a stimulus on the right; and (4) on $25 \%$ there was a stimulus in both positions. Observers made two separate responses (one for the left and one for the right position). Independently, one of the two positions was cued. Observers were told the proportion of the four kinds of trials, and that the cue was not correlated with the presence of a stimulus.

For the low-contrast stimuli, if the noninformative cue attracted attention and therefore made the stimuli higher in contrast and more visible, one would predict that $d^{\prime}$ would be higher in the cued location than in the noncued location; in other words, the proportions of hits $[p$ (hits)] would be higher, and the proportion of false alarms $[p(\mathrm{FA})]$ would be lower in the cued location. On the other hand, if the consequence of the noninformative cue were to affect observer's bias, the criterion to respond "present" should be lower in the cued location; in other words, both $p$ (hits) and $p(\mathrm{FA})$ should be higher in the cued location. We ran the high-contrast group in order to obtain detection performance with the stimuli used in Experiment 2, but we did not expect the cue to have any effect, since performance would be near ceiling.

\section{Method}

The sequence of stimulus events was the same as in Experiments 1 and 2 . On each trial, there could either be 0,1 , or 2 stimuli, as described above. Observers used the keyboard keys " $X$ " and " $Z$," respectively, to indicate whether the left position contained a Gabor stimulus or not. They used the "." and "/" keys, respectively, to indicate whether the right location contained a Gabor stimulus or not. Observers could enter the responses in any order. They were encouraged to take their time and be as accurate as they could. Unlike in the previous experiments (and in Carrasco et al., 2004), observers were given trial-by-trial feedback. When they erred, a computerized voice said, "left wrong" and/or "right wrong."

Each observer participated in at least 2 practice blocks of 48 trials each, and then 3 blocks of 192 trials. The orientation of the Gabor stimulus was randomly determined on each trial. When a Gabor stimulus was present, it was the same contrast as the standard from Experiment 1 for one group of subjects, and the standard from Experiment 2 for the other group. Observers were informed of the proportion of present and absent trials, and also that the cue was not informative as to the presence of a Gabor stimulus. Only peripheral cues were used.

\section{Results and Discussion}

The results are presented in Table 3 . For the low-contrast group, observers' sensitivity, as measured by $d^{\prime}$, was significantly lower for the cued location than for the uncued location $\left(d^{\prime}=1.01\right.$ vs. 1.51 , respectively) $[t(11)=3.27, p<$ .05 , two-tailed]. If involuntary attention increased the perceptual contrast of the stimuli, then observers would have been more accurate with the stimuli in the cued location. The reason they were less accurate is discussed below.

We measured observers' criterion, $C$, as follows:

$$
C=-.5[Z(\text { hits })+Z(\mathrm{FA})] .
$$

Observers had a much less stringent criterion for the cued location in comparison with the uncued location, 36 versus 1.00 , and this difference was reliable $[t(11)=4.00$, $p<.05]$. In other words, the cue increased both hits and FA in the same direction, indicating a shift in bias.

Considering the criterion results, it is not surprising that observers were actually less accurate (measured by $d^{\prime}$ ) for the cued than for the uncued location. If on some trials, observers mistook the cue for the Gabor stimulus (since they were both visual transients), or assumed that something must have been in that location, the FA rate would have been driven up for the cued location, driving down $d^{\prime}$. The FA rate was three times higher for the cued than for the uncued location (Table 3 ).

The results for the high-contrast group are not very remarkable. The cue had no significant effect on $d^{\prime}$ or criterion. The sensitivity for the cued and uncued locations were $d^{\prime}=5.06$ and 5.17 , respectively $[t(5)=0.75$, n.s. $]$. The corresponding criterion measures for the cued and uncued locations were $C=-.09$ and .03 , respectively $[t(5)=1.11$, n.s. $]$. This lack of difference is expected, since observers were nearly perfect in performance. We included the high-contrast group in the detection task only to verify that the stimuli in Experiment 1 were near threshold, those in Experiment 2 being clearly above threshold.

Several readers have expressed surprise that the hit rates were so low for the low-contrast stimuli given that the peak contrasts ranged from .17 to .37 (Table 1), suggesting there must be an error in our contrast measurements. We were extremely careful in measuring the stimulus luminance values. In fact, our results are not surprising, for two reasons. First, as discussed above, peak contrast is a poor way to represent contrast with Gabor stimuli, since it represents the value of only a few pixels and does not take into account exposure duration or the area subtended by the stimulus (Watson, 2000; Watson \& Ahumada, 2005). Second, the threshold of Gabor stimuli increases with eccentricity (Foley et al., 2007; Pointer \& Hess, 1989; Robson \& Graham, 1981). For example, Foley et al. reported for Gabor stimuli that log thresholds increased linearly at a rate of $0.47 \mathrm{~dB}$ for each one-wavelength step in eccentricity. Thus, expectations of

Table 3

Results of Experiment 3

\begin{tabular}{lcccc}
\hline & $p$ (hits) & $p(\mathrm{FA})$ & $d^{\prime}$ & $C$ \\
\hline \multicolumn{5}{c}{ Low Contrast } \\
Cued & .549 & .233 & 1.01 & .36 \\
Uncued & .423 & .073 & 1.51 & 1.00 \\
& \multicolumn{5}{c}{ High Contrast } \\
Cued & .995 & .010 & 5.06 & -.09 \\
Uncued & .994 & .005 & 5.17 & .03 \\
\hline
\end{tabular}

Note-C, criterion 
detection thresholds with sine wave stimuli presented at the fovea do not apply to Gabor stimuli briefly presented in the periphery. In this experiment, it is clear that observers were near detection threshold with the standard stimulus used in Experiment 1, and above threshold with the standard stimulus used in Experiment 2. We replicated Carrasco et al. (2004) with the low-contrast stimuli (Experiment 1), but consistent with Schneider (2006), we did not replicate Carrasco et al. in Experiment 2.

Experiment 3 sheds light on three theories of the effect of involuntary attention on the perception of contrast. Carrasco et al. (2004) claimed that involuntary attention increases contrast. Schneider (2006) demonstrated that this only occurred for stimuli near threshold. He claimed that the cued stimulus would be more likely to be detected, and hence it would be judged to have higher contrast. One construal of this hypothesis is that involuntary attention increases detectability $\left(d^{\prime}\right)$, and this was not the case. Finally, we hypothesized that the results reflect a shift in criterion to report that something had been presented in the cued location, and that is consistent with the present results. ${ }^{7}$

\section{EXPERIMENT 4}

Experiment 3 demonstrated that observers could be biased to believe that a Gabor stimulus was in the cued location. However, a detection task is different from the task used in Experiments 1 and 2 and by Carrasco et al. (2004), where observers were asked the orientation of the Gabor stimulus with higher contrast. Hence, in Experiments 4 and 5 we returned to the original paradigm.

Our explanation of the results of Carrasco et al. (2004) and Schneider (2006) is that observers have a nonperceptual bias to believe that a Gabor stimulus appeared in the cued location. One way to compare an explanation based on perceptual processes with one based on nonperceptual processes is to reverse the timing of the cue and target (Liu et al., 2005; Prinzmetal et al., 2005; Woodman et al., 2003). If the cue comes after the stimulus, it cannot have any effect on the perception of the stimulus. Rather, effects from a cue that appears after the stimulus must reflect nonperceptual processes.

\section{Method}

We ran two versions of this experiment. In Experiment 4A, the procedure was similar to Experiment 1 (time-limit group), except that the cue appeared $50 \mathrm{msec}$ after the target disappeared. As in the previous experiments, the cue duration was $67 \mathrm{msec}$. In Experiment $4 \mathrm{~B}$, there were two conditions, randomly intermixed. On half of the trials, the cue appeared $50 \mathrm{msec}$ before the target (a replication of Experiment 1), and on the other half, the cue appeared $300 \mathrm{msec}$ after the target disappeared. There were 12 observers in each experiment. In both experiments, we used the low-contrast stimuli that we used in Experiment 1. In all other respects, these experiments were identical to Experiment 1 (without the center cue condition).

\section{Results and Discussion}

Experiment 4A. The proportion of trials on which observers responded with the test Gabor stimulus as a function of whether the test stimulus or the standard stimulus was cued is shown in Figure 6. As in Experiment 1, observ-

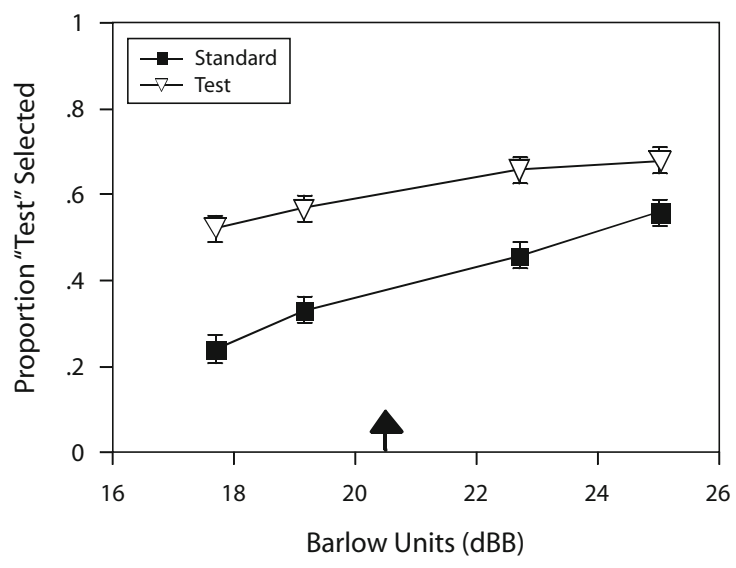

Figure 6. Experiment 4A: The proportion of trials on which observers responded that the test stimulus had greater contrast than the standard stimulus, as a function of which item was cued (standard or test). Note that the cue appeared after the stimulus. The arrow marks the contrast of the standard.

ers were more likely to respond that the test stimulus had greater contrast when it was cued, despite the fact that the cue appeared $50 \mathrm{msec}$ after the target was removed. Observers were significantly more likely to respond that the test stimulus had greater contrast when it was followed by a cue than when it was not $[F(1,11)=32.49, p<.01]$.

Orientation judgments were affected by the cue, but not in a manner expected by an attentional account of the results. When the location was correct, observers were significantly less likely to get the orientation correct when the higher contrast item was cued than when it was not cued $(72.8 \%$ vs. $78.2 \%$, respectively) $[t(11)=2.86, p<.05]$. When the location judgment was incorrect, orientation judgments were near chance, as would be expected (see Table 2).

Experiment 4B. Figure 7 compares the proportions of trials on which observers responded with the test Gabor pattern when the cue preceded the target and when it followed the target by $300 \mathrm{msec}$. The pattern of results is very similar. There was a significant cuing effect for when the cue preceded the target $[F(1,11)=10.12, p<.01]$ and when the cue followed the target by $300 \mathrm{msec}[F(1,11)=$ $47.80, p<.01]$

One might be tempted to argue that perception takes place over time and that perceptual analysis was still occurring when the cue appeared; thus, the cue might still affect perception. However, the tendency to respond with the test stimulus when it was cued was actually slightly larger when the cue followed the stimulus than when it preceded the stimulus. With the cue preceding the target, the proportion of trials on which the test was selected was .555 when the test was cued and .456 when the standard was cued. These proportions when the cue followed the target were .541 and .451 for the test and standard cued, respectively. The interaction of ISI and which item was cued (test or standard) was significant $[F(1,11)=21.08, p<.01]$. If the effect of the cue was on the target perception, it should have a larger effect when it preceded the cue. 
Cue 50 msec Before Target

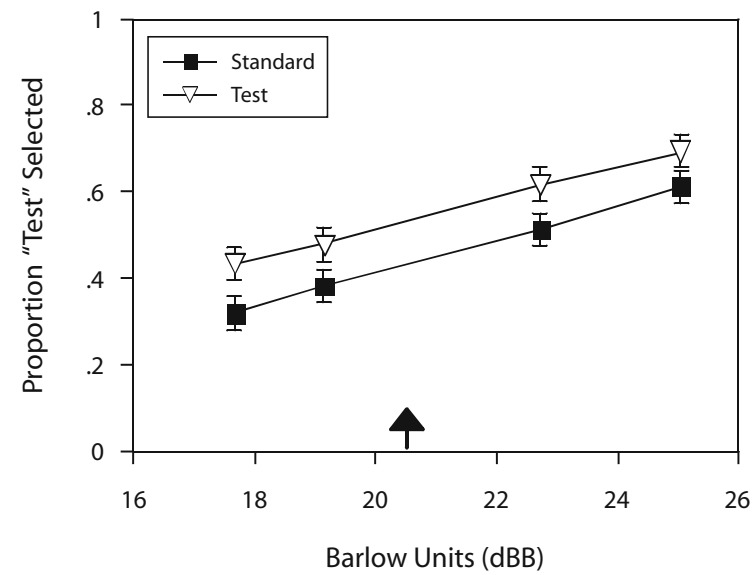

Cue 300 msec After Target

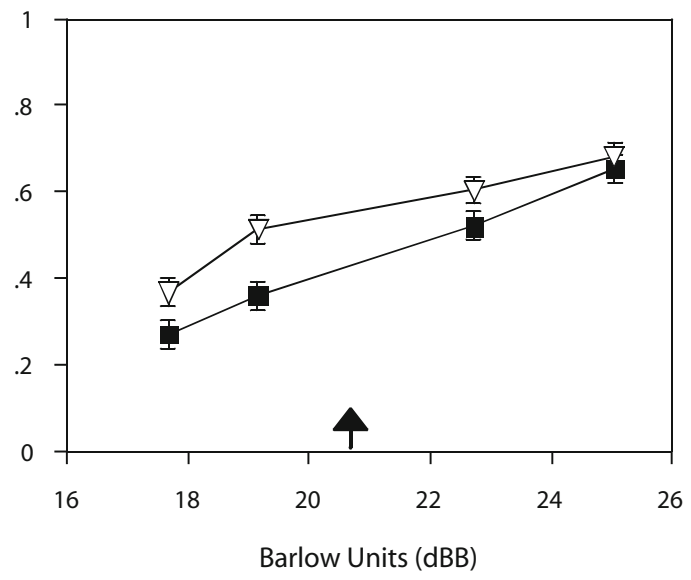

Figure 7. Experiment 4B: The proportion of trials on which observers responded that the test stimulus had greater contrast than the standard stimulus, as a function of which item was cued (standard or test). In the left panel, the cue preceded the stimulus by $50 \mathrm{msec}$, and in the right panel it followed the stimulus by $300 \mathrm{msec}$. The arrows mark the contrast of the standard.

The manipulation of having the cue after the stimulus has been used to discriminate perceptual and nonperceptual processes (e.g., Liu et al., 2005; Prinzmetal et al., 2005; Woodman et al., 2003). For example, Woodman et al. argued that if one obtains a cuing effect with the cue following the target, it might be that the cue is affecting retrieval from visual working memory. Another way to interpret results with the cue appearing after the stimulus is in terms of location uncertainty: Observers do not know which location to base their response on, and they are biased to resolve this uncertainty by basing their response on the cued location. This does not mean that the perceptual representation of the stimulus is changed by the cue. Rather, there is a bias to report what observers think occurred in the cued location.

Once again, orientation judgments failed to show a positive effect of the cue. When observers got the location incorrect, they got the orientation correct on $47 \%$ of the trials, which is close to chance. However, when they got the location correct, they were correct on the orientation on $75.9 \%$ of the trials. As in Experiments 1 and 4A, orientation judgments were significantly less accurate when the higher contrast item was cued than then it was not cued (73.5\% vs. $78.2 \%$, respectively) $[t(11)=2.86, p<.05]$. This finding reinforces our interpretation that on some trials observers do not perceive the Gabor stimuli. If they have a bias to report that there was something in the cued location, then they will guess the orientation of the stimulus in that location, even though they did not perceive a Gabor stimulus there. Such guesses would be at chance, lowering the accuracy of location judgments when the higher contrast item was in the cued location. We do not know why we sometimes find that observers are significantly less accurate for location judgment in the cued than the uncued location (Table 2). However, we have found no cases where observers are more accurate on orientation judgment for stimuli in the cued location.

\section{EXPERIMENT 5}

In Experiment 5, we tested our hypothesis that the previous results were due to a bias by testing how observers would respond when there was no Gabor stimulus in either position. The experiment was precisely like Experiment 1, with a time-limit group; except on $20 \%$ of the trials on which data were collected, there were no Gabor stimuli in the display. Increasing the gain on nothing should result in nothing. Hence, if the spatial cue is increasing contrast, on the target-absent trials there is no reason for observers to select the cued location over the uncued location. However, if the visual transient of the cue biases observers to think that there was a Gabor stimulus in the cued location, they should be more likely to respond that the cued location had higher contrast even though there was nothing in that location.

This experiment was inspired by the observation that some observers complained in Experiments 1 and 4 that on some trials, they did not perceive anything. In a sense, the target-absent trials play the same role as in the detection experiment: They reveal nonperceptual biases.

\section{Method}

The experiment was identical to Experiment 1, except for the 20\% of trials on which no Gabor stimulus was presented. This fact was not mentioned to the observers. Seventeen observers participated.

\section{Results and Discussion}

The critical results in this experiment are the responses on trials on which (unbeknownst to observers) there was no Gabor stimulus. On these trials, observers selected the cued location as having higher contrast than the uncued location $(62.1 \%$ vs. $37.9 \%)$ even though there was nothing in either location. Sixteen of 17 observers were more likely to select the cued location as having higher contrast than the uncued location. Assuming that the probability of selecting the cued location was $50 \%$, this was significantly greater than chance $[t(16)=5.63, p<.01]$. 
Results from the target-present trials are shown in Figure 8. As in the previous experiments, observers were significantly more likely to respond that the test stimulus had higher contrast when it was cued than when the standard stimulus was cued $[F(1,16)=32.42, p<.01]$.

We examined performance on orientation judgments when the location was correct. Like the previous experiments, the orientation judgments were not more accurate for the cued than for the uncued location (see Table 2). The accuracy on the orientation judgments when the location was correct was not influenced by the cue $(66.3 \%$ for cued and $66.3 \%$ for noncued trials). When the location judgment was incorrect, accuracy in the cued and uncued locations were $47.6 \%$ versus $49.6 \%$, respectively. On the target-present trials, observers performed less well in this experiment (the psychometric functions are not as steep) perhaps because observers became more discouraged by the difficulty of performing the task when, unbeknownst to them, occasionally no Gabor stimulus was presented.

\section{GENERAL DISCUSSION}

In Experiment 1, we replicated the results of Carrasco et al. (2004). Observers were more likely to respond that a cued stimulus had higher contrast than an uncued stimulus. In Experiment 2, we increased the contrast of the stimuli and the effect disappeared, consistent with Schneider (2006). One interpretation of these results is that involuntary or automatic attention increases the contrast of low-contrast stimuli, but has little effect on high-contrast stimuli.

We presented an alternative hypothesis: The tendency to select the cued location results from a nonperceptual bias. With very low-contrast stimuli, observers may believe, while not perceiving anything in the uncued location, that something appeared in the cued location. We tested this in three additional ways. Experiment 3 was a detection experiment and observers had to make independent decisions about the presence of a Gabor stimulus in each posi-

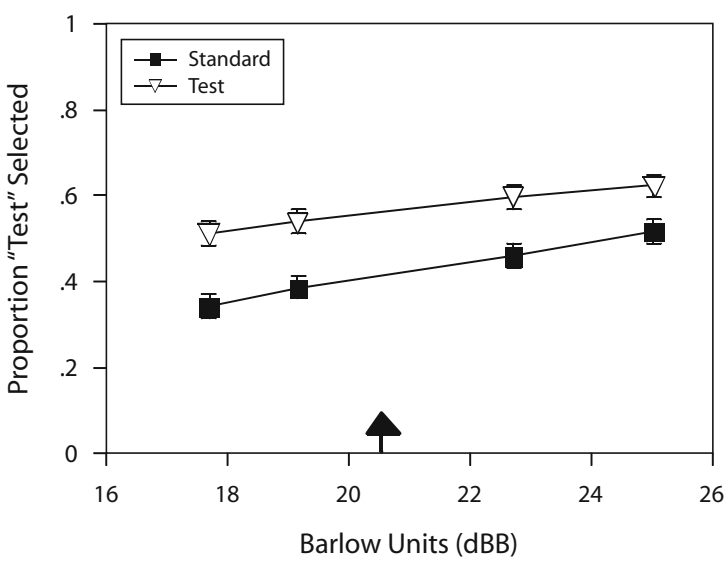

Figure 8. Experiment 5: On Gabor-present trials, the proportion of trials on which observers responded that the test stimulus had greater contrast than the standard stimulus, as a function of which item was cued (standard or test). The arrow marks the contrast of the standard. tion. The measure of detectability, $d^{\prime}$, was actually lower for the cued location than for the noncued location. On the other hand, the criterion to report that a stimulus was present was lower in the cued location. The cue increased hit rate but also substantially increased false alarm rate.

In Experiment 4, we returned to the procedure used by Carrasco et al. (2004). However, in the critical conditions, the cue appeared after the Gabor stimuli. The results were the same as in the first experiment: Observers were more likely to report that the stimulus in the cued location had higher contrast than did the uncued stimulus. Postcuing procedures have been used to compare perceptual and nonperceptual processes with the argument that if the cue appears after the stimulus has disappeared, it does not have an opportunity to affect the perception of the stimulus. Even if the processing of the stimulus extends over time, one would expect that a perceptual effect of the cue would be larger when it precedes the stimulus than when it follows the stimulus, and this was not the case.

Experiment 5 was a replication of the Experiment 1, except that on $20 \%$ of trials no stimulus was present. On these stimulus-absent trials, observers consistently selected the cued stimulus as having greater contrast than the uncued stimulus.

Our explanation of the cuing effects reported by Carrasco et al. (2004) and Schneider (2006) is that the results are due to a bias to report that something appeared in the cued location. Carrasco et al. did acknowledge and test a possible bias account of their data, but their bias explanation is different from ours. They tested, and rejected, the possibility that observers would have a tendency to respond with the cued location regardless of the task. Thus, when asked which location had less contrast, observers still had a tendency to respond with the uncued location. Our explanation is consistent with this finding. Suppose on a given trial, the stimuli are at or below threshold, so that the observer does not have a strong impression of a stimulus at either location. If the left location is cued, the observer might believe that there was a stimulus at that location, either because there was a visual transient in that location (the cue) or because they lowered their criterion (in signal detection terms) for that location. If one then asked which location had less contrast, it would have to be the right location, because the observer thought there was something in the left location, but not in the right location.

Consistent with our interpretation is that orientation judgments were never more accurate when the Gabor with the higher contrast appeared in the cued location (see Table 2). This finding is curious because we know that voluntary attention improves orientation perception (e.g., Prinzmetal et al., 1998). If the nonpredictive cue is enhancing perception, it should enhance orientation perception as well as contrast. We never found this to be the case. Carrasco et al. (2004) did not report orientation judgment accuracy.

The method used by Carrasco et al. (2004), Schneider (2006), and Tsal et al. (1994, Experiment 4) of presenting two stimuli and asking which had greater contrast is open to bias explanations at several levels. Observers might have a tendency to respond with the cued location (or avoid responding with the cued location) for a variety 
of nonperceptual reasons. The attentional cue might affect which stimulus the observer responds to, without affecting the perceptual representation of that stimulus. This problem has been called location uncertainty. A number of investigators have been concerned that the results of attention experiments reflect processing of the target stimulus as opposed to not knowing which location contained the target (e.g., Luck, Hillyard, Mouloua, \& Hawkins, 1996; Shiu \& Pashler, 1994).

The problem of location uncertainty is not limited to low-contrast stimuli. Consider a recent study by Cameron, Tai, and $\mathrm{Carrasco}^{8}$ (2002; cf. Liu et al., 2005). Observers were presented with a Gabor stimulus that was rotated slightly clockwise or counterclockwise from vertical (the target). Observers had to indicate the orientation of the target. They may have been uncertain as to which location contained the target. If they were biased to respond with whatever information was in the cued location on trials when the distractor was cued, performance would have been worse than when the target was cued, because the observer was basing the response on the wrong stimulus. Finding that orientation judgments were more accurate when the target was cued does not mean that the cue changed the perceptual representation; rather, it may mean that observers have a bias to believe that the target was in the cued location..$^{9}$ Observers are responding with the information in the cued location, but that does not mean that the information in that location is enhanced.

To test this hypothesis, Gould, Wolfgang, and Smith (2007; cf. Smith, 2000) performed an experiment similar to Carrasco et al. (2004), but with two conditions. In one condition there was location uncertainty. In the other condition, the target location was marked with high-contrast lines that indicated the target location ("fiducial markers"). They found that the observers were more accurate on the cued than on the uncued location only when the fiducial markers were not present.

Whenever this paradigm is used, various kinds of bias and location uncertainty are present. Schneider and Komlos (in press) demonstrate that even when the stimuli are above threshold, the results from using this paradigm are subject to decision bias. A safer method is to have one stimulus and ask observers to judge the brightness or contrast of that single stimulus against a standard. Furthermore, one should ensure that observers know which location contained the target (e.g., Prinzmetal et al., 1997). Using this more standard spatial cuing paradigm, without location uncertainly, it is possible to have accuracy higher in the cued location, but only with an informative spatial cue (e.g., Prinzmetal et al., 2005).

There are many cases in the literature where voluntary or endogenous attention makes perception more accurate even when there is clearly no location uncertainty (e.g., Luck et al., 1996; Prinzmetal et al., 1998; Prinzmetal et al., 2005). These are cases where the allocation of attention is goal-directed: It is strategically advantageous to allocate perceptual processing resources to a particular location or object. For example, in the spatial cuing tasks, voluntary attention has been manipulated by a cue spatially informative cue. In these cases, attention improves performance by increasing the accuracy of perception. Thus voluntary attention does alter perception.

One might ask: What of the observation by Ebbinghaus (1908) and others that a faint stimulus (e.g., the ticking of a watch) is not detected unless one is attending to the stimulus? There are several examples in the literature where a stimulus would have been detectable in terms of luminance energy, yet because the observer was not attending, it was undetected (e.g., Mack, 2003; Mack \& Rock, 1998; Rensink, O’Regan, \& Clark, 2000). Attention can influence detectability. Energy contrast can increase detectability. However, it does not follow that attention increases phenomenal contrast. Such an argument would be equivalent to claiming "Windsurfing makes me happy. Doing research makes me happy. Therefore, windsurfing makes me work" (or "work makes me windsurf"). Unfortunately, these activities cannot be done at the same time.

The difference between the effect of attention on detectability and phenomenal contrast might explain the paradox pointed out by James (1890). James thought that if one does not attend to a faint stimulus, it may not be perceived. Yet, if we are aware of a stimulus, attention does not seem to increase its intensity. We know today that attention may limit access to consciousness of stimuli that would otherwise have sufficient stimulus intensity to be perceived.

There is little doubt that voluntary attention does alter the appearance of objects so that our perception of what we are attending to is more veridical (e.g., Prinzmetal et al., 1998; Prinzmetal et al., 1997; Prinzmetal \& Wilson, 1997). Attention should make our interactions with the world more successful, and it can only do this by making our perceptions more accurate, not by shifting them. We have called this idea attentional constancy (e.g., Prinzmetal et al., 1997; Prinzmetal et al., 1998). Like lightness constancy, attention should lead to a more veridical perception of the world. Consider the task of a mammal foraging for ripe fruit. Most mammals do not have color perception. It might be that the ripe fruit (e.g., red tomatoes) and unripe fruit (green tomatoes) can be discriminated on the basis of brightness contrast. The ripe fruit might have higher contrast with the background than the unripe fruit. As the animal scans the environment, if attention changed contrast, unripe fruit might appear to be ripe fruit. To be a useful mechanism in an evolutionary sense, attention should not lead us astray.

\section{AUTHOR NOTE}

This research was based, in part, on an honors thesis by V.L. at the University of California, Berkeley. We thank Jeffrey Tsai, Beibei Luo, Jonathan Hirschberg, and Deena Elwan for their help running the experiments, and Sam Ling for providing the software used by Carrasco et al. (2004). We also thank Keith Schneider and Philip Smith for their valuable discussion of the issues, and Andrew Watson for advice on describing contrast. Michael Esterman, Psyche Louie, Ayelet Landau, and Christin McCool made invaluable comments on the manuscript. Correspondence concerning this article should be addressed to W. Prinzmetal, Psychology Department, University of California, Berkeley, CA 94720 (e-mail: wprinz@berkeley.edu).

\section{REFERENCES}

Beard, B. L., \& Ahumada, A. J., JR. (1999). Detection in fixed and random noise in foveal and parafoveal vision explained by template learning. Journal of the Optical Society of America A, 16, 755-763. 
Cameron, E. L., Tai, J. C., \& Carrasco, M. (2002). Covert attention affects the psychometric function of contrast sensitivity. Vision Research, 42, 949-967.

Carrasco, M., Ling, S., \& Read, S. (2004). Attention alters appearance. Nature Neuroscience, 7, 308-313.

Ebiinghaus, H. (1908). Psychology: An elementary text-book (M. Meyer, Trans. \& Ed.). Boston: Heath.

Foley, J. M., Varadharajan, S., Koh, C. C., \& Farias, M. C. Q. (2007). Detection of Gabor patterns of different sizes, shapes, phases and eccentricities. Vision Research, 47, 85-107.

GARDNER, G. T. (1973). Evidence for independent parallel channels in tachistoscopic perception. Cognitive Psychology, 4, 130-155.

Gould, I. C., Wolfgang, B. J., \& Smith, P. L. (2007). Spatial uncertainty explains exogenous and endogenous attentional cuing effects in visual signal detection. Journal of Vision, 7(13, Art. 4), 1-17.

Hoffman, J. E. (1979). A two-stage model of visual search. Perception \& Psychophysics, 25, 319-327.

Hoffman, J. E., Nelson, B., \& Houck, M. R. (1983). The role of attentional resources in automatic detection. Cognitive Psychology, 15, $379-410$.

James, W. (1890). The principles of psychology (Vol. 2). New York: Holt.

Ling, S., \& CARrasco, M. (2007). Transient covert attention does alter appearance: A reply to Schneider (2006). Perception \& Psychophysics, 69, 1051-1058.

Liu, T., Pestilli, F., \& Carrasco, M. (2005). Transient attention enhances perceptual performance and fMRI response in human visual cortex. Neuron, 45, 469-477.

Loftus, G. R., \& Masson, M. E. J. (1994). Using confidence intervals in within-subject designs. Psychonomic Bulletin \& Review, 1, 476-490.

LucK, S. J., Hillyard, S. A., Mouloua, M., \& Hawkins, H. L. (1996). Mechanisms of visual-spatial attention: Resource allocation or uncertainty reduction? Journal of Experimental Psychology: Human Perception \& Performance, 22, 725-737.

MACK, A. (2003). Inattentional blindness: Looking without seeing. Current Directions in Psychological Science, 12, 180-184.

MACK, A., \& Rock, I. (1998). Inattentional blindness. Cambridge, MA: MIT Press.

PoINTER, J. S., \& Hess, R. F. (1989). The contrast sensitivity gradient across the human visual field: With emphasis on the low spatial frequency range. Vision Research, 29, 1133-1151.

Posner, M. I. (1980). Orienting of attention. Quarterly Journal of Experimental Psychology, 32, 3-25.

Posner, M. I., SnYder, C. R. R., \& Davidson, B. J. (1980). Attention and the detection of signals. Journal of Experimental Psychology: General, 109, 160-174

Prinzmetal, W. (2005). Location perception: The X-Files parable. Perception \& Psychophysics, 67, 48-71.

Prinzmetal, W., Amiri, H., Allen, K., \& Edwards, T. (1998). Phenomenology of attention: 1 . Color, location, orientation, and spatial frequency. Journal of Experimental Psychology: Human Perception \& Performance, 24, 261-282.

Prinzmetal, W., \& Banks, W. P. (1983). Perceptual capacity limits in visual detection and search. Bulletin of the Psychonomic Society, 21, 263-266.

Prinzmetal, W., McCool, C., \& Park, S. (2005). Attention: Reaction time and accuracy reveal different mechanisms. Journal of Experimental Psychology: General, 134, 73-92.

Prinzmetal, W., Nwachuku, I., Bodanski, L., Blumenfeld, L., \& Shimizu, N. (1997). The phenomenology of attention: 2. Brightness and contrast. Consciousness \& Cognition, 6, 372-412.

Prinzmetal, W., \& Wilson, A. (1997). The effect of attention on phenomenal length. Perception, 26, 193-205.

Rensink, R. A., O'Regan, J. K., \& Clark, J. J. (2000). On the failure to detect changes in scenes across brief interruptions. Visual Cognition, 7, 127-145.

Robson, J. G., \& Graham, N. (1981). Probability summation and regional variation in contrast sensitivity across the visual field. Vision Research, 21, 409-418.
SCHNeIDER, K. A. (2006). Does attention alter appearance? Perception \& Psychophysics, 68, 800-814.

SCHNeIDER, K. A., \& Komlos, M. (in press). Attention biases decisions but does not alter appearance. Journal of Vision.

ShiU, L.-P., \& PASHLER, H. (1994). Negligible effect of spatial precuing on identification of single digits. Journal of Experimental Psychology: Human Perception \& Performance, 20, 1037-1054.

SмiтH, P. L. (2000). Attention and luminance detection: Effects of cues, masks, and pedestals. Journal of Experimental Psychology: Human Perception \& Performance, 26, 1401-1420.

Tsal, Y., Shalev, L., ZaKay, D., \& Lubow, R. E. (1994). Attention reduces perceived brightness contrast. Quarterly Journal of Experimental Psychology, 47A, 865-893.

Warner, C. B., JuOla, J. F., \& Koshino, H. (1990). Voluntary allocation versus automatic capture of visual attention. Perception \& Psychophysics, 48, 243-251.

WATSON, A. [B.] (2000). Visual detection of spatial contrast patterns: Evaluation of five simple models. Optics Express, 6, 12-33.

Watson, A. B., \& AhUmada, A. J., JR. (2005). A standard model for foveal detection of spatial contrast. Journal of Vision, 5, 717-740.

Watson, A. B., Barlow, H. B., \& Robson, J. G. (1983). What does the eye see best? Nature, 302, 419-422.

Woodman, G. F., Vecera, S. P., \& Luck, S. J. (2003). Perceptual organization influences visual working memory. Psychonomic Bulletin \& Review, 10, 80-87.

\section{NOTES}

1. James (1890) notes several examples where he believed that attention makes a sensation "stronger than it would be otherwise" (p. 425); but he noted paradoxically "the intensification brought about by attention never seems to lead us astray" (p. 426).

2. Tsal et al. (1994) had observers memorize numbers to correspond to different gray levels. Consider a particular gray stimulus on a black background. That stimulus would appear brighter than it was. If during practice observers adjust their responses to be as accurate as possible, they adjust the response categories accordingly. They would do this more on easy blocks (with attention) and difficult blocks (without attention). Prinzmetal et al. (1997) replicated Tsal et al. when observers responded with numbers, but not when they responded in a matching task.

3. In each experiment, there was a way to objectively demonstrate that the stimuli were above threshold. For example, in many experiments there were catch trials without a stimulus. Observers were always nearly perfect at detecting catch trials.

4. The software used in these experiments will be archived at www .neuroexpt.com/.

5. We generated the stimuli in this manner because in several pilot experiments, in which we generated our own Gabor stimuli, we failed to replicate Carrasco et al. (2004). In retrospect, we think that the stimulus contrast in these pilot experiments might have been too great.

6 . It is sum of the squared contrast at each pixel $\times$ the area of the pixel $\left(\right.$ degrees $\left.^{2}\right) \times$ time.

7. Both the increased detectability and criterion-shifting hypothesis are consistent with Schneider (2006)

8. Cameron et al. (2002) compared performance with $100 \%$ predictive cues and neutral cues. They argued that they were studying involuntary "transient" attention because the time between the onset of the cue and the onset of the target was rather short $(121 \mathrm{msec})$. However, with practice, observers can allocate voluntary attention within $50 \mathrm{msec}$ (Warner, Juola, \& Koshino, 1990). Hence, it is unclear whether their effects should be attributed to voluntary attention, involuntary attention, or both.

9. Liu et al. (2005) included a condition with the cue appearing after the display as a control. Unfortunately, the cue-after condition showed the same pattern of results, in terms of accuracy, as the cue-before condition. The difference was not reliable in the cue-after condition. However, the lack of significant difference should not be taken as definitive, since there were only 6 observers.

(Manuscript received June 26, 2007; revision accepted for publication March 28, 2008.) 